\title{
Aditivos para hormigones, morteros y pastas Normativa: clasificación y definiciones
}

\author{
Prof. Dr. DEMETRIO GASPAR-TEBAR
}

IETCC/CSIC

\begin{abstract}
$R E S U M E N$
En el presente artículo se estudia la clasificación y definiciones de los aditivos que se utilizan en la fabricación de hormigones, morteros y pastas hechos a base de cementos, objeto y campo de aplicación de la norma UNE 83-200-84 (editada en diciembre de 1984), elaborada por el Grupo de Trabajo n. 2 "Aditivos" de la Comisión Técnica n. 83 "Hormigón" del IRANOR.

Por otra parte, se realiza un estudio de la normativa y documentos relacionados con la clasificación y definiciones de los aditivos de carácter internacional, así como de los países de tecnología avanzada.
\end{abstract}

\begin{abstract}
$S U M M A R Y$
In this work, classification and definitions of the additives used to produce concretes, mortars and plasters which base is cement, aim and application field of the UNE 83-200-84 norm (edited on December 1984) elaborated by Working Group n. 2 "Additives" of the IRANOR Technical Commission n. 83 "Concretes", are studied.

On another hand, it is carried out a study of the normative and of the documents related with the classification and the definitions of the additives of international feature, so as those of advanced technology' countries.
\end{abstract}

\section{INTRODUCCION}

En un trabajo anterior (1) se dió cuenta de la importancia que ha adquirido el empleo de los aditivos en la fabricación de hormigones, morteros y pastas, así como de las modificaciones y efectos especificos que producen, lo que ha motivado la necesidad de contar con una normativa española apropiada, cuya realización se encomendó a un Grupo de Trabajo (GT-2 "Aditivos") creado, al efecto, de la CT-83 "Hormigón" del IRANOR.

En el presente artículo, que precede a otros, se estudia la clasificación y definiciones de los aditivos que se utilizan en la elaboración de hormigones, morteros y pastas hechos a base de conglomerantes hidráulicos (cementos); clasificación y definiciones que se han adoptado en la norma española UNE 83-200-84 (diciembre 1984) de reciente aparición. Esta norma, como objetivo prioritario, ha sido preparada por el mencionado Grupo de Trabajo (GT-2 "Aditivos") de la CT-83 "Hormigón" del IRANOR (*).

(*) En la actualidad forman parte de este Grupo de Trabajo (CT-83/GT-2):

Demetrio Gaspar Tébar; IETCC (Presidente)._Gabriel Fernández Fernández; Asland, S. A. (Vicepresidente).--José R. Dominguez. Bidagor; Halesa (Secretario).-Manuel Alvarez Losada; Consejo Superior de Aparejadores.-Carlos Chacón Barrilero; ANI._José M." Fernández Paris, INTEMAC.-Pedro Ferré Franquet; Sinor-Kao, S. A.-José M." Garcia San Martin; Seopan.-Antonio Garrido Hernández; Laboratorio de Ensayos, Colegio Oficial de Aparejadores y Arquitectos Técnicos de Murcia.-Gerardo de Lucas Pérez; Grace, S. A.-Gloria Martin Vallejo; INCE.-M." José Monsalve de la Asunción; Laboratorio d'Assaigs Coll. d'Aparelladores i Arq. Técnics de Tarragona._Javier de Nicolás Caparrós: ANL.-Gustavo Pezzi Peñalver: Halesa.-Rafael Sassot Iribarren: ANI.-Gabriel Sémelas Ledesma; Master Builders.—Jesús Serra: TEXA.-Jesủs Temiño Fernández; CELTITE, S. A. 


\section{DEFINICION DE ADITIVOS PARA HORMIGONES, MORTEROS Y PASTAS SEGUN UNE 83-200-84}

\subsection{Definición de aditivos}

Según la norma UNE 83-200-84, aditivos son aquellas sustancias o productos que incorporados al hormigón, mortero o pasta antes o durante el amasado y/o durante un amasado suplementario, en una proporción no superior al $5 \%$ del peso de cemento (salvo casos especiales), producen la modificación deseada en dicho hormigón, mortero o pasta en estado fresco y/o endurecido, de alguna de sus caracteristicas, de sus propiedades habituales o de su comportamiento.

En el apartado 3 "DEFINICION DE ADITIVOS" de dicha norma se advierte:

a) Que el empleo de un aditivo no debe deteriorar las características mecánicas, quimicas o físicas del hormigón, mortero o pasta, aún cuando, eventualmente, se puede aceptar una pequeña disminución de dichas características; así mismo, el empleo de un aditivo no debe alterar las características de las armaduras, tanto del hormigón armado como pretensado.

b) Que cuando se utilicen varios aditivos, éstos tienen que ser compatibles entre sí y, además, se debe conocer la influencia mutua en los resultados finales.

c) Que los aditivos (excepto los sólidos poco solubles en agua) se deben incorporar disueltos en el agua de amasado o en forma de dispersión estable, después de mezclarlos y homogeneizarlos con toda o con una parte de dicha agua de amasado; no se deben incorporar al cemento, a no ser que dicha incorporación se tenga que efectuar de acuerdo con las características propias del aditivo, de la fabricación del hormigón o que figure, explícitamente, en las prescripciones especiales del mismo, o de la obra.

De acuerdo con la definición de aditivos un reductor de agua de amasado que, además, puede modificar el tiempo de fraguado (acelerándolo, por ejemplo) en unas dosis determinadas, se debe comercializar como tal reductor de agua de amasado y no como acelerador de fraguado, a pesar de que dicho aditivo posea dos funciones de distinta categoría que se pueden aprovechar beneficiosamente.

\subsection{Funciones de los aditivos}

De acuerdo con el criterio adoptado en la norma UNE 83-200-84, para definir a los aditivos, cada uno de ellos se caracteriza por producir una modificación determinada $-\mathrm{y}$ solamente unade alguna de la propiedades o caracteristicas del hormigón, mortero o pasta en estado fresco o endurecido; propiedad que se define en dicha norma como FUNCION PRINCIPAL. Por otra parte, los aditivos pueden modificar accesoriamente alguna, o algunas, de las propiedades $\mathrm{o}$ caracteristicas del hormigón, mortero o pasta en estado fresco o endurecido, independientemente de la que define a la función principal; esta propiedad se define como FUNCION O FUNCIONES SECUNDARIAS.

Además, los aditivos pueden producir otras modificaciones inevitables de ciertas propiedades o características de los hormigones, morteros o pastas, que no se requieren como función secundaria (por ejemplo, débil descenso de las resistencias mecánicas, retraso del tiempo de fraguado, etc.), propiedad que se define como EFECTO O EFECTOS SECUNDARIOS, en cuyo caso el fabricante del aditivo lo debe poner en conocimiento del usuario.

La eficacia de las funciones mencionadas de cada aditivo depende del tipo de hormigón, de la dosificación de dicho hormigón, de los materiales utilizados en la fabricación del hormigón, de las condiciones de puesta en obra, etc. 


\section{CLASIFICACION Y DEFINICION DE LOS ADITIVOS, SEGUN UNE 83-200-84}

Definida la función principal de los aditivos como la modificación, y solo una modificación, que producen de las características o propiedades del hormigón, mortero o pasta en estado fresco o endurecido, permite (dicha definición) la clasificación de los diversos productos o sustancias que se pueden utilizar como tales aditivos; clasificación que, a título de ejemplo, se cita a continuación, en donde se incluye una relación de productos o sustancias agrupadas de acuerdo con la modificación que pueden producir en la reologia de los hormigones, morteros o pastas (aumentando la trabajabilidad para una relación agua/cemento dada o reduciendo la cantidad de agua de amasado para una consistencia o trabajabilidad determinada); en los tiempos de fraguado y endurecimiento; en el contenido de aire o de otros gases; en la durabilidad química, física o mecánica y en otras propiedades:

\section{Productos o sustancias que modifican la reología de los hormigones, morteros o pastas:}

\section{I.1. Mejoran la trabajabilidad:}

- Alginatos; bentonitas; cales grasas; caseína; cenizas volantes; diatomeas (harina fósil); puzolanas molidas, etc.

\section{I.2. Reducen la cantidad de agua de amasado para tener una consistencia dada:}

- Abietatos alcalinos; ácidos hidroxycarboxílicos, sales de; calcio, lignosulfonatos de; hidrocarburos sulfonados, sales de; ester de poliglicol; polisacáridos de bajo peso molecular; sodio, lignosulfonatos de; sulfonato de alkilarilo; etc., que reducen dicha cantidad de agua en cantidades iguales o superiores al $3 \%$.

- Condensados sulfonados de melamina - formaldehído; condensados sulfonados de naftalenoformaldehído; lignosulfonatos modificados y otros tipos que reducen la cantidad de agua en cantidades iguales o superiores al $12 \%$.

II. Productos o sustancias que modifican el fraguado y/o el endurecimiento de los hormigones, morteros $\mathbf{y}$ pastas:

- Acido cítrico; ácidos glucónicos; ácidos hidroxicarbosílicos, sales de los; ácidos lignosulfónicos, sales de los alcalinos, hidróxidos; almidón; alunita; azúcares; borax; calcio, cloruro de; fluoruros; fluosilicato de sodio; calcio, formiato de; calcio, nitrato de; cinc, óxidos de; fosfatos; glicerina; glucosa; hidratos de carbono (glucosa-sacarosa); lignosulfonatos; magnesio, sales de; plomo, óxido de; polisacáridos de bajo peso molecular; sacarosa; sodio, aluminato de; trietanolamina; etc.

III. Productos o sustancias que modifican el contenido de aire o de otros gases en los hormigones, morteros o pastas:

- Acido abiético, sales del; ácido primérico, sales del (neutralizado con resinas de madera); ácidos grasos y sus sales; alkilarilsulfonatos; alkilsulfatos; calcio, cloruro de; detergentes; etanolamina, sales de; fenoletoxilatos; jabones; lignosulfitos; lignosulfonatos; resina vinsol; sodio, estearato de; sulfonatos de alcoholes grasos; trietanolamina; etc. que pueden actuar como inclusores de aire.

- Agua oxigenada más hipoclorito de calcio; aluminio, polvo de; calcio, carbonato ácido de, 
más ácido clorhídrico; calcio, carburo de; cinc, polvo de; hierro, polvo de; polvos metálicos oxidables (hierro, aluminio, cinc, etc.); resinas vinílicas-sulfonatos alkilarilicos (estabilizador); sodio, benzoato de (estabilizador de burbujas); etc. que pueden generar gases y espumas.

\section{Productos o sustancias que mejoran la resistencia a las acciones físicas:}

- Alcoholes, varias formas de; etilen-glicol; acelerante más inclusor de aire más dispersante; etc. que se pueden utilizar como anticongelantes.

- Acidos grasos, sales de los; aluminio, sulfato de; fluidificantes; sodio, alginatos de; sodio, carbonato de; sodio, oxalato de; etc. que se pueden usar como repulsores de agua $\mathrm{o}$ hidrófugos.

\section{Productos o sustancias que mejoran la resistencia a las acciones fisicoquímicas:}

- Lechada de cemento; fosfatos; potasio, cromato de; potasio, molibdato de; sodio, benzoato de; sodio, cromato de; sodio, molibdato de; sodio, nitrito de; etc., que se pueden emplear como inhibidores de corrosión de armaduras.

Por todo ello, y de un modo indirecto, se han normalizado las funciones principales que han dado lugar a la clasificación de los aditivos que se incluyen en la Tabla 1, según que modifiquen la reología, el fraguado y/o el endurecimiento, el contenido de aire o de otros gases, que generen expansión, que. mejoren la durabilidad o que actuen sobre otras propiedades o características de los hormigones, morteros y pastas elaborados a base de cemento, cuyas funciones principales son:

a) Mejorar la trabajabilidad de los hormigones, morteros o pastas para una determinada relación agua/cemento o permitir la reducción de la cantidad de agua de amasado para una trabajabilidad dada; generalmente, estos aditivos son sólidos finamente divididos (ADITIVOS PLASTIFICANTES).

b) Disminuir la cantidad de agua de amasado de un hormigón, mortero o pasta (en cantidades iguales o superiores al $5 \%$ para los dos primeros y al $3 \%$ para el tercero) para una trabajabilidad dada o, por ampliación, aumentar notablemente la trabajabilidad para una misma cantidad de agua; también, con estos aditivos, se pueden obtener simultáneamente ambos fenómenos (ADITIVOS REDUCTORES DE AGUA O FLUIDIFICANTES).

c) Aumentar, significativamente, la trabajabilidad de un hormigón, mortero o pasta para una relación agua/cemento dada o reducir considerablemente la cantidad de agua de amasado (en cantidades iguales o superiores al $12 \%$ ) para una trabajabilidad determinada; como en el caso anterior, también se pueden obtener simultáneamente,

TABLA 1

Clasificación de los aditivos, según la norma

UNE 83 - 200 - 84

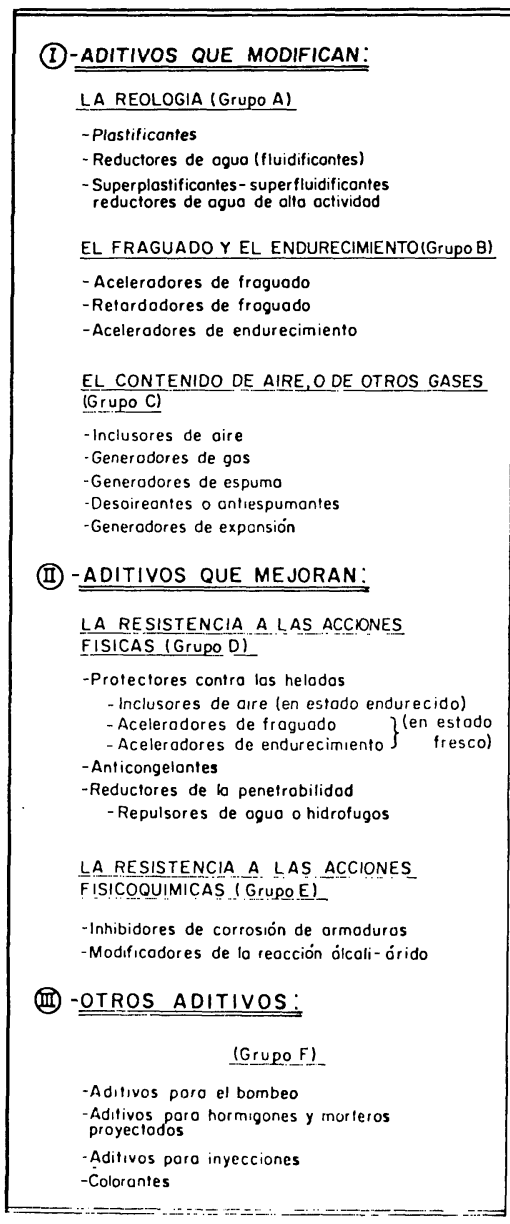


con estos aditivos, ambos fenómenos (ADITIVOS SUPERPLASTIFICANTES, SUPERFLUIDIFICANTES O REDUCTORES DE AGUA DE ALTA ACTIVIDAD).

d) Reducir o adelantar el tiempo de fraguado del cemento (principio y final) que se encuentra en el hormigón, mortero o pasta (ADITIVOS ACELERADORES DE FRAGUADO).

e) Retrasar el tiempo de fraguado (principio y final) del cemento que se encuentra en el hormigón, mortero o pasta (ADITIVOS RETARDADORES DE FRAGUADO).

f) Aumentar o acelerar el desarrollo de las resistencias mecánicas iniciales de los hormigones, morteros o pastas (ADITIVOS ACELERADORES DE ENDURECIMIENTO).

g) Producir en los hormigones, morteros o pastas, un número elevado de finas burbujas de aire, separadas y repartidas uniformemente, que permanecen asi durante el endurecimiento de los materiales aditivados (ADITIVOS INCLUSORES DE AIRE).

h) Producir un gas por medio de una reacción quimica durante la colocación del hormigón, mortero o pasta (ADITIVOS GENERADORES DE GAS).

i) Producir, por medios mecánicos, una espuma estable formada por burbujas de aire de tamaño variable, distribuidas homogéneamente dentro de la masa, a la que confiere una estructura alveolar (ADITIVOS GENERADORES DE ESPUMA).

j) Eliminar el exceso de aire introducido en la masa del hormigón, mortero o pasta por el empleo de ciertos áridos o de ciertos aditivos utilizados para obtener otra función principal (ADITIVOS DESAIREANTES O ANTIESPUMANTES).

k) Producir una expansión controlada y permanente en los hormigones, morteros y pastas (ADITIVOS GENERADORES DE EXPANSION).

1) Mejorar la resistencia a las heladas de los hormigones, morteros o pastas (ADITIVOS PROTECTORES CONTRA LAS HELADAS).

m) Disminuir el punto de congelación del agua de amasado; además, pueden impedir la aparición de cristales de hielo en el hormigón, mortero o pasta (ADITIVOS ANTICONGELANTES).

n) Incrementar la resistencia al paso del agua bajo presión a través de hormigones, morteros o pastas endurecidas (ADITIVOS QUE REDUCEN LA PENETRABILIDAD DEL AGUA).

ñ) Disminuir la capacidad de absorción capilar o la cantidad de agua que pasa a través de un hormigón, mortero o pasta, saturado y sometido a un gradiente hidráulico (ADITIVOS HIDROFUGOS O REPULSORES DE AGUA).

o) Reducir la posibilidad de corrosión de las armaduras embebidas en el hormigón o mortero (ADITIVOS INHIBIDORES DE CORROSION DE ARMADURAS).

p) Impedir o dificultar la reacción entre ciertos áridos y los álcalis del cemento, asi como reducir sus efectos expansivos (ADITIVOS MODIFICADORES DE LA REACCION ALCALI-ARIDOS).

q) Reducir el rozamiento externo del hormigón, mortero o pasta contra la tuberia de conducción, sin modificar la relación agua/cemento (ADITIVOS PARA EL BOMBEO). 
r) Mejorar las condiciones de proyección al disminuir el descuelgue del material proyectado y el rechazo (ADITIVOS PARA HORMIGONES Y MORTEROS PROYECTADOS).

s) Aumentar la fluidez de los rellenos o morteros (de inyección) y reducir los riesgos de exudación y decantación (ADITIVOS PARA INYECCIONES).

t) Colorear al hormigón, mortero o pasta (ADITIVOS COLORANTES).

\section{DOCUMENTACION CONSULTADA}

Para realizar la mencionada norma UNE 83-200-84 se han consultado las normas y documentos de carácter internacional, así como de aquellos paises de tecnología avanzada, que se citan a continuación:

\subsection{Documentos y nor- mas europeos}

4.1.1. Rapport final del Grupo de Trabajo RILEM "Aditivos", del año 1967, que clasifica y define a los aditivos de acuerdo con la acción o efecto principal del aditivo (equivale a la función principal) sobre los morteros y hormigones en los 9 Grupos, denominados agentes, de la Tabla 2 , según que modifiquen la reología, el contenido de aire, el fraguado y endurecimiento, asi como el color de los morteros y hormigones; que generen expansión de dichos morteros y hormigones y que mejoren la resistencia a las acciones fisicas, mecánicas, químicas y biológicas. En cada grupo se incluyen los aditivos correspondientes, definidos en dicho rapport y que se reseñan en la mencionada Tabla 2.

4.1.2. Norma belga NBN 805-01, de mayo de 1969, que por una parte define al aditivo en general $y$, por otra, clasifica y define a los aditivos de acuerdo con la modificación que producen en los morteros u hormigo-
TABLA 2

Aditivos: Clasificación

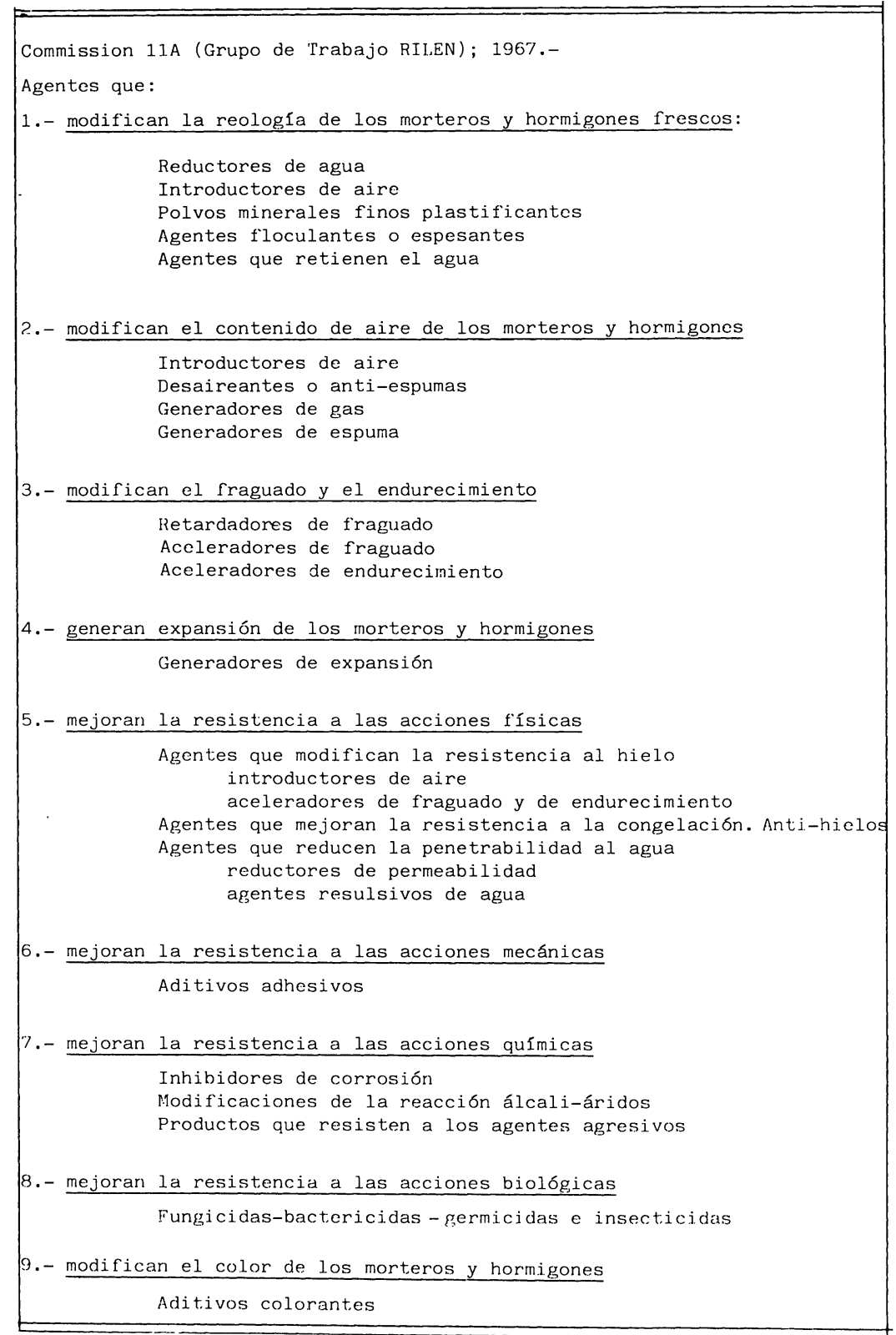


nes; modificación que se considera como "acción principal". La clasificación de los aditivos de esta norma, que se incluye en la Tabla 3, coincide esencialmente con la realizada por el Grupo de Trabajo RILEM “Aditivos", mencionada en el apartado anterior y que figura en la Tabla 2; así mismo, las definiciones de los distintos aditivos, aunque más simplificadas en la norma presente, también coinciden en gran parte con las efectuadas por el mencionado Grupo de Trabajo RILEM “Aditivos”.

4.1.3. Norma italiana UNI 7101, de octubre de 1980, que define a los aditivos en general y los clasifica en 9 grupos de acuerdo con la acción principal que ejercen sobre pastas, morteros y hormigones; dicha clasificación figura en la Tabla 4. La clasificación de esta norma incluye a los aditivos fluidificantes-aireantes, flidificantes-retardadores y fluidificantes-acelerantes, que se denominan de acción mixta.

4.1.4. Normas británicas BS 5075: part 1: 1982; BS 5075: part 2: 1982 y BS 1014, que tienen por objeto especificar las características y métodos de ensayo para los aditivos que se pueden utilizar con el fin de modificar una o varias propiedades del hormigón relacionadas con la trabajabilidad, con el fraguado y endurecimiento y con las resistencias mecánicas, en la

T ABLA 3

Aditivos: Clasificación según normas

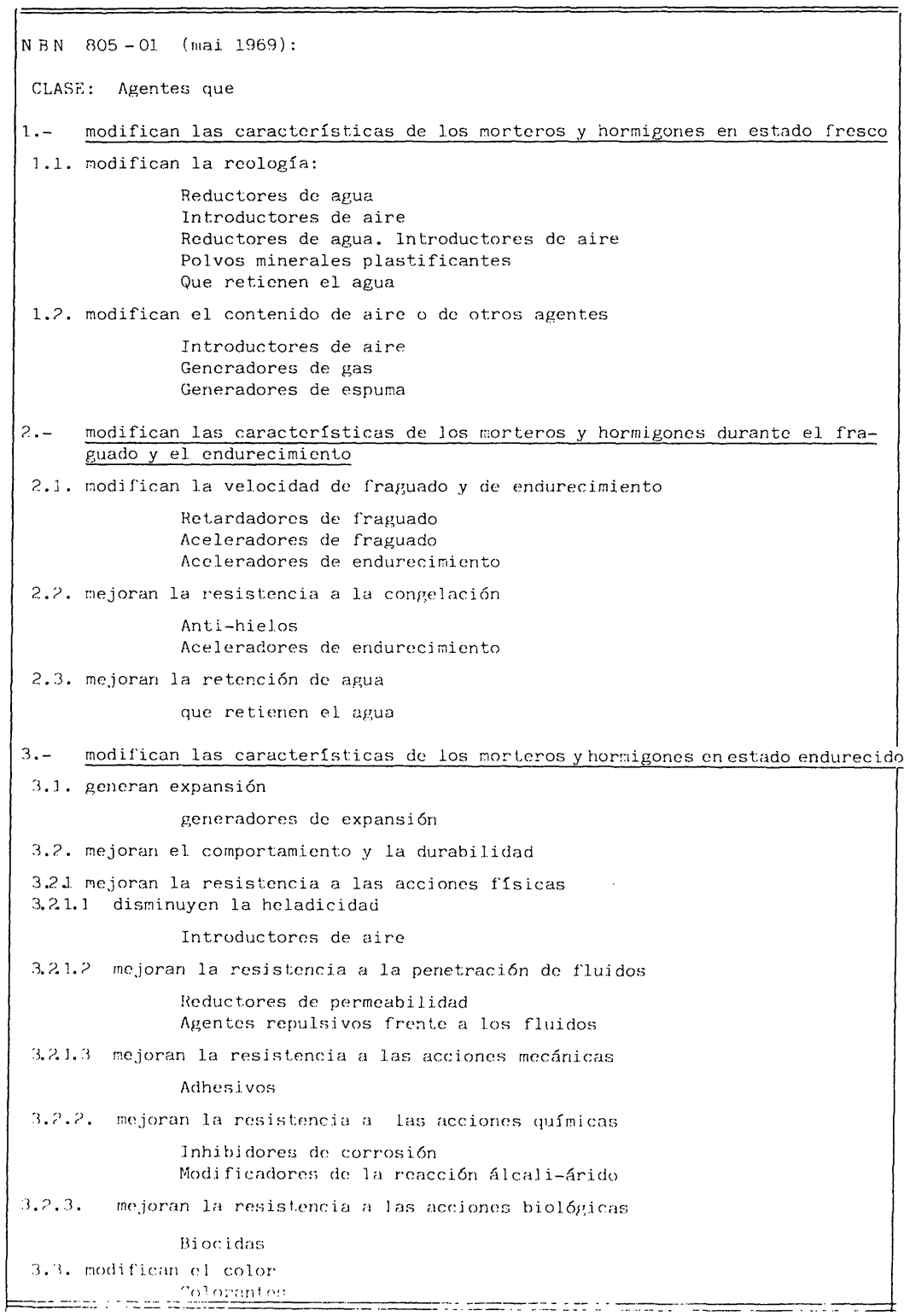
primera, con el contenido de aire en la segunda y con el color en la tercera; aditivos que figuran en la Tabla 4.

La norma BS 5075: part 1: 1982 clasifica a los aditivos en 5 categorías, incluyendo (entre ellos) a los reductores de agua-acelerantes y reductores de agua-retardadores, que combinan dos funciones (reducción de agua y modificación del tiempo de fraguado, acelerándolo o retardándolo).

4.1.5. Norma francesa NF P18-103, de noviembre de 1983, que define a los aditivos para hormigones, morteros y pastas, elaborados a base de conglomerantes hidráulicos, establece una clasificación y precisa su marcado. La clasificación de los aditivos, que se incluye en la Tabla 4, se basa en su función principal (y solo una) de dichos aditivos, caracterizada por la o las modificaciones que introduce en las propiedades del hormigón, mortero o pasta en estado fresco o endurecido. 
En esta clasificación se incluye el grupo de aditivos fluidificantes, cuya función principal (según la norma mencionada) es la de producir un aumento importante de la trabajabilidad de la mezcla, señalando por otra parte que ciertos fluidificantes se pueden utilizar como reductores de agua-plastificantes, en cuyo caso sus condiciones de empleo deben ser las de éstos. Dichos aditivos fluidificantes se corresponden, en gran parte, con los superfluidificantes de otras normas.

La función principal de los reductores de agua-plastificantes es, para una misma trabajabilidad, producir un aumento de las resistencias mecánicas como consecuencia de una reducción del contenido de agua del hormigón, mortero o pasta.

\subsection{Documentos y normas de Estados Unidos}

4.2.1. Norma ASTM C 494-84, que cubre a los materiales que se emplean como aditivos químicos para ser añadidos en las mezclas de hormigón de cemento portland con objeto de reducir la cantidad de agua de amasado para conseguir una consistencia dada (Tipo A), retrasar el fraguado del cemento en el hormigón (Tipo B), acelerar el fraguado y el desarrollo de las resistencias mecánicas iniciales del hormigón (Tipo C), reducir la cantidad de agua de amasado para una consistencia dada como los aditivos del tipo A y retrasar el fraguado del cemento en el hormigón como los aditivos del tipo B (Tipo D), reducir la cantidad de agua de amasado para una consistencia dada como los aditivos del tipo A y acelerar el fraguado y el desarrollo de las resistencias mecánicas iniciales del hormigón como los aditivos del tipo $\mathrm{C}$ (Tipo E), reducir la cantidad de agua de amasado que se necesita para conseguir una consistencia dada en una cantidad igual o superior al $12 \%$ (Tipo F) y reducir la cantidad de agua de amasado como los aditivos del tipo $\mathrm{F}$ y retrasar el tiempo de fraguado del cemento en el hormigón como los aditivos del tipo B (Tipo G).

La clasificación de los aditivos que se hace en esta norma figura en la Tabla 4, en donde se incluyen tres tipos de aditivos polifuncionales (Tipo D, Tipo E y Tipo G).

4.2.2. Admixtures for Concrete; Reported by ACI, Committee 212 (Documento ACI 212, 1 R-81), que clasifica a los aditivos en los cinco grupos principales que figuran en la Tabla 4. En el grupo quinto "miscelánea" se incluyen 12 categorías de aditivos, de acuerdo con los efectos o modificaciones que producen en las características del hormigón.

TABLA 4

Aditivos: Clasificación según normas

\begin{tabular}{|c|c|}
\hline UNI 7101 (ottobre 1980).- & ASTM C $494-82 .-$ \\
\hline $\begin{array}{l}\text { - Fluidificante } \\
\text { - Aireante } \\
\text { - Retardador } \\
\text { - Acelerante } \\
\text {. Fluidificante - aireante } \\
\text { - Fluidificante - retardador } \\
\text { - Fluidificante - acelerante } \\
\text { - Antihielo } \\
\text { - Superfluidificante }\end{array}$ & \multirow[t]{2}{*}{$\begin{array}{l}\text { Tipo Aditivos } \\
\text { A.- Aditivos raductores de agua } \\
\text {. B.- Aditivos retardadores } \\
\text {. C.- Aditivos acclorantes } \\
\text {. D.- Aditivos reductores de agua y retardado } \\
\text {. E.- Aditivos reductores de agua y res } \\
\text { acelerantes } \\
\text {. F.- Aditivos reductores de agua de } \\
\text { alta actividad } \\
\text {. G.- Aditivos reductores de agua de alta } \\
\text { actividad y retardadores }\end{array}$} \\
\hline BS 5075: Part 1: 1982.- & \\
\hline \multirow{2}{*}{$\begin{array}{l}\text { En apartado "3. Definitions", } \\
\text { - Acelerante } \\
\text { - Retardador } \\
\text { - Reductor normal de agua } \\
\text { - Reductor de agua acelerante } \\
\text { - Reductor de agua retardador }\end{array}$} & $\begin{array}{c}\text { COMITE } 212 \text { (ACI 2.12.1R-81) "Aditivos para el } \\
\text { hormigon" }\end{array}$ \\
\hline & \multirow{6}{*}{$\begin{array}{l}\text { - Acelerantes } \\
\text { - Introductores de airc } \\
\text { - Reductores de agua y reguladores de } \\
\text { fraguado } \\
\text { - Aditivos mincrales finamente divididos } \\
\text { - Miscelánea: } \\
\text { - Generadores de gas } \\
\text { - Aditivos para inyecciones } \\
\text { - Generadores de expansión } \\
\text { - I,igantes } \\
\text { - Aditivos nara bombeo (ayuda) } \\
\text { - Colorantes } \\
\text { - Floculantes } \\
\text { - Fungicidas, germicidas e insecticidas } \\
\text { - Impermeabilizantes } \\
\text { - Reductores de permeabilidad } \\
\text { - Aditivos químicos para reducir la } \\
\text { - expansion álcali-árido } \\
\text { - Inhibidores de corrosion }\end{array}$} \\
\hline - Inclusores de aire & \\
\hline BS 1014 & \\
\hline . Pigmentos & \\
\hline NF $\quad \mathrm{P} 18-103$ (novembre 1983) & \\
\hline $\begin{array}{l}\text { - Acelerador de fraguado sin cloruros } \\
\text { - Acelerador de endurecimiento sincloruros } \\
\text { - Fluidificantes } \\
\text { - Hidrofugo de masa } \\
\text { - Plastificante } \\
\text { - Reductor de agua - plastificante } \\
\text { - Introdador de fraguado } \\
\text { - Aditivo para pastas de inyección en el } \\
\text { - Generador de gas } \\
\text { - Generador de espuma } \\
\text { - Colorante pormon pretensado } \\
\text { - Aditivo para hormigon omortero proyectadol }\end{array}$ & \\
\hline
\end{tabular}


TABLA 5

Aditivos: Clasificación según normas

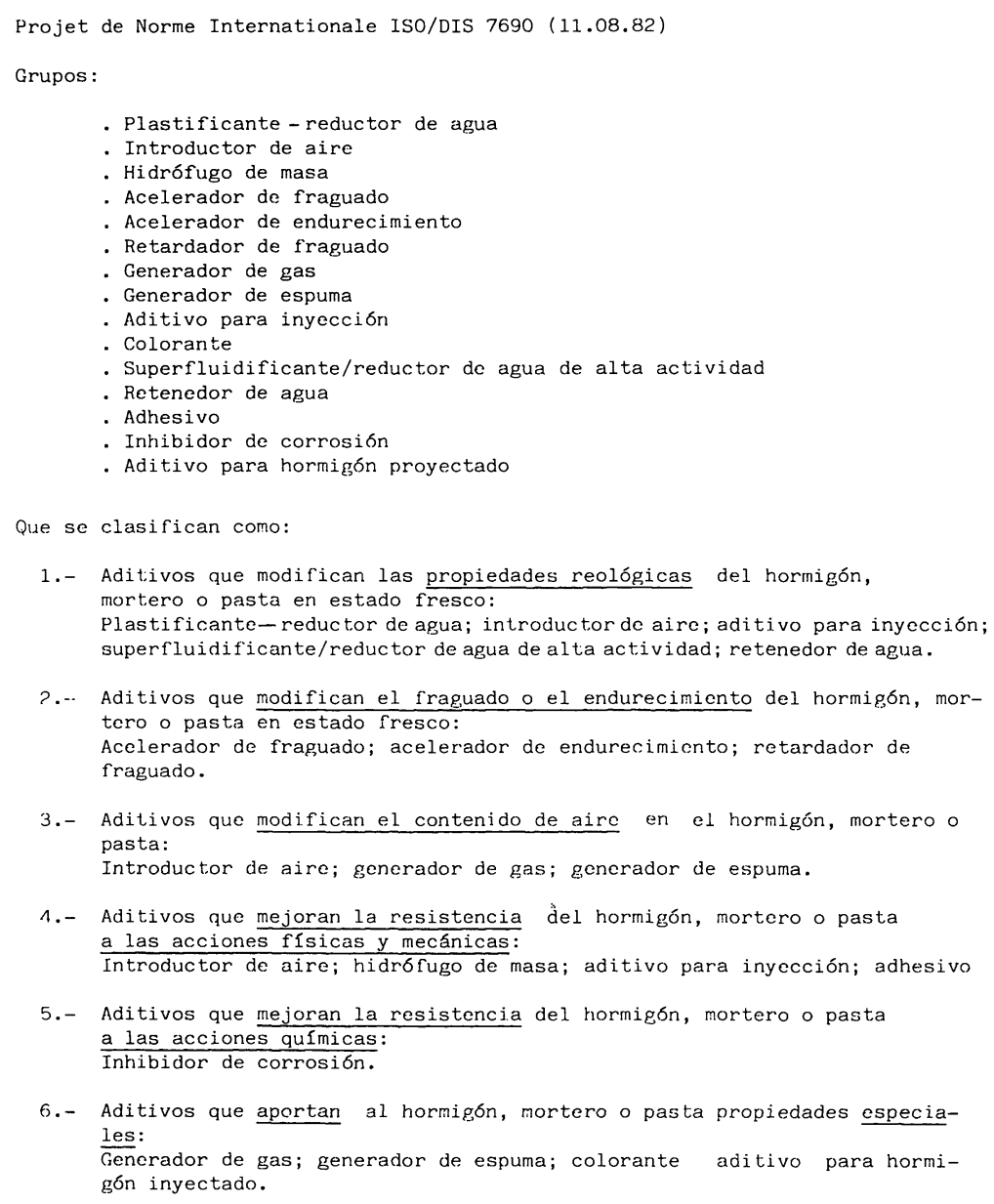

b) Mejoren la resistencia del hormigón, mortero o pasta a las acciones físicas, mecánicas y quimicas.

c) Aporten al hormigón, mortero o pasta propiedades especiales.

\section{BIBLIOGRAFIA}

(1) GASPAR-TEBAR, D. (1984): Aditivos para hormigones, morteros y pastas. Materiales de Construcción; julio-agostoseptiembre, 195, 31-40.
4.3. Normas internacionales

4.3.1. Projet de Norme Internationale ISO/DIS 7690 , sometido a votación el 11 de febrero de 1982, que define a los aditivos para hormigones, morteros y pastas hechos a base de conglomerantes hidráulicos y establece, además, una clasificación. Cada aditivo está definido por la o las funciones especificas que le corresponden, caracterizadas por la o las modificaciones mayores que introducen en las propiedades $o$ en el comportamiento de los hormigones, morteros $\mathrm{o}$ pastas.

En la Tabla 5 figuran los aditivos definidos en esta norma y su clasificación en seis grupos según que:

a) Modifiquen las propiedades reológicas, el fraguado o el endurecimiento, o el contenido de aire de los hormigones, morteros y pastas.

(Continuará) 